CRYSTALLOGRAPHIC COMMUNICATIONS

ISSN 2056-9890

Received 27 May 2020

Accepted 16 July 2020

Edited by L. Fabian, University of East Anglia, England

Keywords: peroxosolvates; aliphatic amino acids; charge assisted hydrogen bonds; peroxide $\mathrm{H}$-bonded chains; carboxylate anions; crystal structure.

CCDC reference: 2016846

Supporting information: this article has supporting information at journals.iucr.org/e

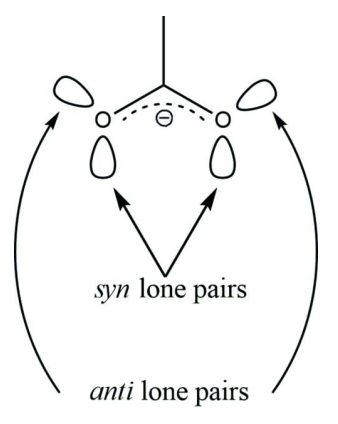

OPEN $\partial$ ACCESS

\section{DL-Piperidinium-2-carboxylate bis(hydrogen peroxide): unusual hydrogen-bonded peroxide chains}

Mger A. Navasardyan, Dmitry A. Grishanov, Petr V. Prikhodchenko and Andrei V. Churakov*

Institute of General and Inorganic Chemistry, Russian Academy of Sciences, Leninskii prosp. 31, Moscow 119991, Russian Federation. ${ }^{*}$ Correspondence e-mail: churakov@igic.ras.ru

The title compound, $\mathrm{C}_{6} \mathrm{H}_{11} \mathrm{NO}_{2} \cdot 2 \mathrm{H}_{2} \mathrm{O}_{2}$, is the richest (by molar ratio) in hydrogen peroxide among the peroxosolvates of aliphatic $\alpha$-amino acids. The asymmetric unit contains a zwitterionic pipecolinic acid molecule and two hydrogen peroxide molecules. The two crystallographically independent hydrogen peroxide molecules form a different number of hydrogen bonds: one forms two as donor and two as acceptor ([2,2] mode) and the other forms two as donor and one as acceptor ([2,1] mode). The latter hydrogen peroxide molecule forms infinite hydrogen-bonded hydroperoxo chains running along the $c$-axis direction, which is unusual for aliphatic $\alpha$-amino acid peroxosolvates.

\section{Chemical context}

Peroxosolvates are crystalline adducts of hydrogen peroxide with various organic or inorganic compounds. Since they are convenient solid sources of active oxygen, some of them have become widely used commercial bleaching, disinfection and oxidation reagents (Jakob et al., 2012; Cronin et al., 2017). It is well known that their stability is strongly dependent on the hydrogen-bonded motifs formed by hydrogen peroxide (Chernyshov et al., 2017). On other hand, $\mathrm{H}_{2} \mathrm{O}_{2}$ is one of the most important signalling molecules in biological systems ( $\mathrm{Li}$ et al., 2020; To et al., 2020). The structures of amino acid peroxosolvates have been studied intensively as simple models of hydrogen peroxide binding with proteins (Prikhodchenko et al., 2011; Kapustin et al., 2014). Peroxide and water-peroxide clusters are now of special interest since they may simulate cooperative hydrogen-bonded switching in the transportation of hydrogen peroxide species through cell membranes (Grishanov et al., 2017; Varadaraj \& Kumari, 2020; Wang et al., 2020). Recently, several structures of organic peroxosolvates with peroxide hydrogen-bonded 1D-aggregates have been reported (Chernyshov et al., 2017; Navasardyan et al., 2017, 2018).

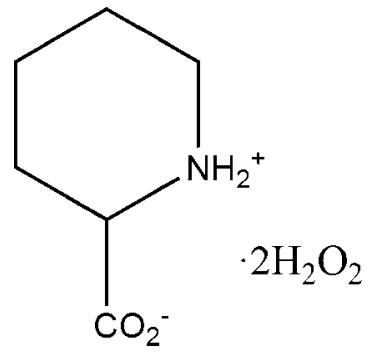




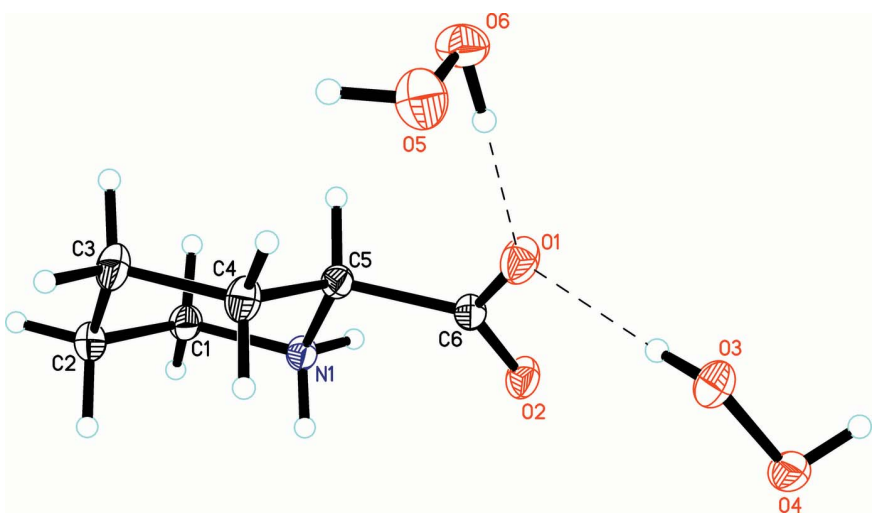

Figure 1

The asymmetric unit of (I) with the atom-numbering scheme. Displacement ellipsoids are drawn at the $50 \%$ probability level. Hydrogen bonds are shown as dashed lines.

\section{Structural commentary}

The asymmetric unit of the title compound (I) comprises a pipecolinic acid molecule and two crystallographically independent peroxide molecules (Fig. 1). As expected, the amino acid coformer exhibits the zwitterionic form with almost equal $\mathrm{C}-\mathrm{O}$ distances [1.2429 (11) and 1.2639 (11) $\AA$ ] $]$. All bond lengths and angles in the organic coformer are close to those observed in the structures of pure pipecolinic acid [(II);

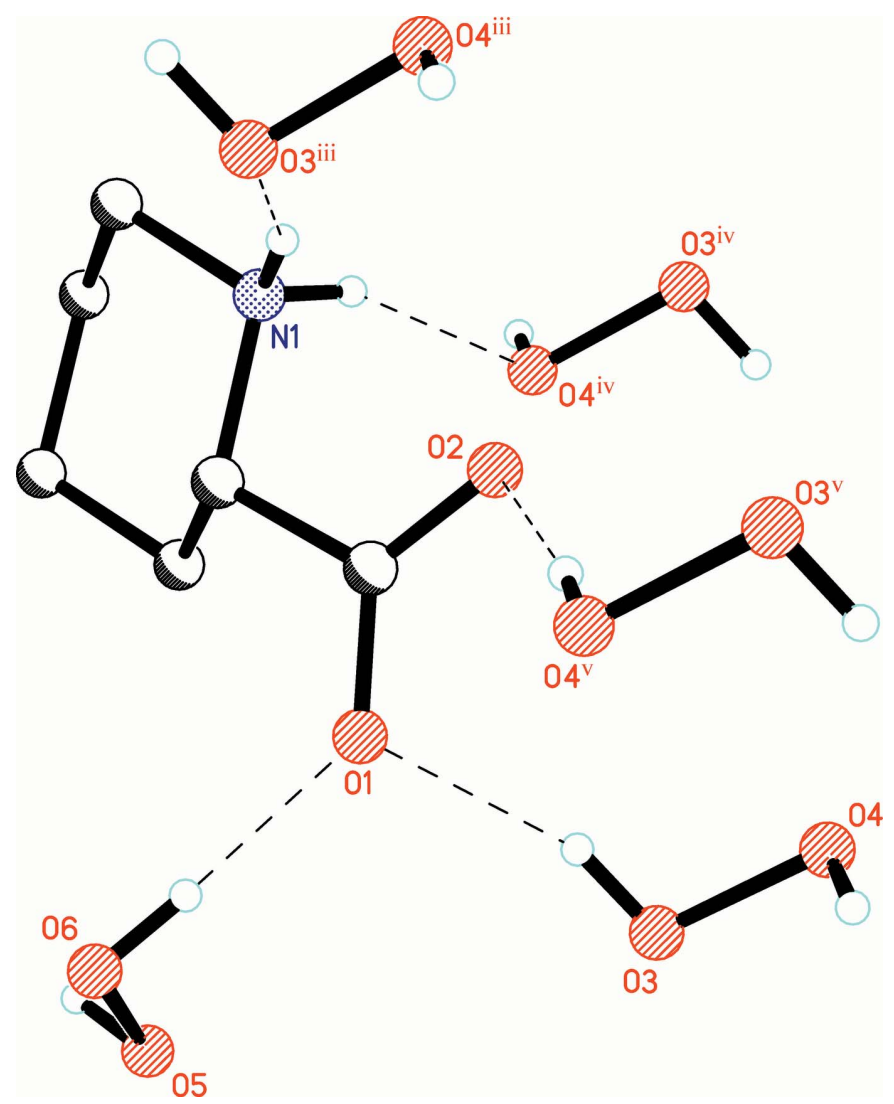

Figure 2

Pipecolinic acid with neighbouring hydrogen-bonded molecules. Hydrogen bonds are shown as dashed lines. [Symmetry codes: (iii) $-1+x, y, z$; (iv) $1-x, 1-y, 1-z$; (v) $1-x, 1-y,-z$.]
Table 1

Hydrogen-bond geometry $\left(\AA,^{\circ}\right)$.

\begin{tabular}{|c|c|c|c|c|}
\hline$D-\mathrm{H} \cdots A$ & $D-\mathrm{H}$ & $\mathrm{H} \cdots A$ & $D \cdots A$ & $D-\mathrm{H} \cdots A$ \\
\hline $\mathrm{O} 3-\mathrm{H} 3 \cdots \mathrm{O} 1$ & $0.872(17)$ & 1.819 (17) & $2.6463(10)$ & $157.8(15)$ \\
\hline $\mathrm{O} 4-\mathrm{H} 4 \cdots \mathrm{O} 2^{\mathrm{i}}$ & $0.866(18)$ & $1.889(18)$ & $2.7490(10)$ & $172.2(15)$ \\
\hline 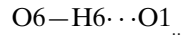 & $0.881(16)$ & $1.760(16)$ & $2.6412(10)$ & $177.4(14)$ \\
\hline $\mathrm{O} 5-\mathrm{H} 5 \cdots \mathrm{O} 6^{\mathrm{ii}}$ & 0.883 (17) & $1.898(18)$ & $2.7777(12)$ & $174.4(15)$ \\
\hline $\mathrm{N} 1-\mathrm{H} 1 \cdots \mathrm{O} 3^{\mathrm{iii}}$ & $0.912(15)$ & $1.961(15)$ & $2.8336(11)$ & $159.6(13)$ \\
\hline $\mathrm{N} 1-\mathrm{H} 2 \cdots \mathrm{O} 4^{\mathrm{iv}}$ & $0.875(15)$ & $2.112(15)$ & $2.9459(11)$ & $159.1(12)$ \\
\hline
\end{tabular}

Symmetry codes: (i) $-x+1,-y+1,-z$; (ii) $x,-y+\frac{3}{2}, z+\frac{1}{2}$; (iii) $x-1, y$, $z$; (iv) $-x+1,-y+1,-z+1$.

Stapleton \& Tiekink, 2001) and pipecolinic acid tetrahydrate [(III); Bhattacharjee \& Chacko, 1979; Lyssenko et al., 2006]. As observed for (II) and (III), the pipecolinic acid molecule in (I) adopts a chair conformation with the carboxylate group occupying the equatorial position. It is of interest to note in all three structures (I), (II), and (III), the core amino acid fragments $\mathrm{N}-\mathrm{C}-\mathrm{CO}_{2}$ are almost planar, with $\mathrm{N}-\mathrm{C}-\mathrm{C}-\mathrm{O}$ torsion angles of less than $22^{\circ}$. This is obviously caused by electrostatic interactions between the oppositely charged amino and carboxylic groups.

\section{Supramolecular features}

In the crystal, the organic molecule acts as a donor of two $\mathrm{N}^{+}-\mathrm{H} \cdots \mathrm{OHOH}$, and as an acceptor of three $\mathrm{COO}^{-} \cdots \mathrm{HOOH}$ hydrogen bonds (Table 1, Fig. 2). The $\mathrm{O}-\mathrm{O}$ bond lengths $[1.4600(9)$ and $1.4646(11) \AA]$ are typical for amino acid peroxosolvates (mean value of $1.465 \AA$ according to the latest, March 2020 version of the CSD; Groom et al., 2016). Both crystallographically independent peroxide molecules occupy general positions and adopt skew conformations with $\mathrm{H}-\mathrm{O}-\mathrm{O}-\mathrm{H}$ torsion angles of $102.5(15)$ and $-105.1(15)^{\circ}$. It is well known that peroxide molecules always form at least two donor hydrogen bonds in the structures of organic peroxosolvates (Chernyshov et al., 2017) and

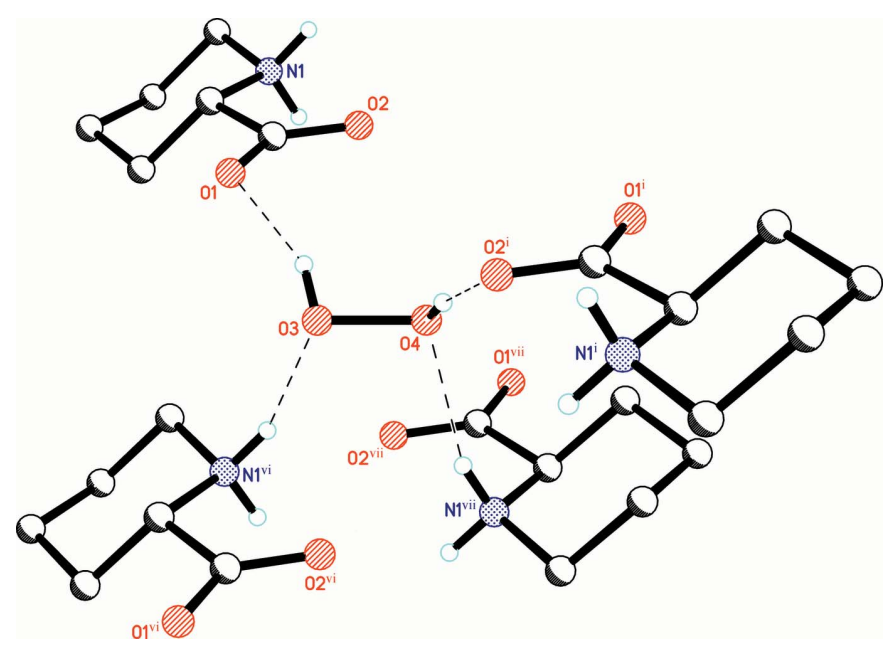

Figure 3

Hydrogen bonds formed by the peroxide molecule $\mathrm{H} 3-\mathrm{O} 3-\mathrm{O} 4-\mathrm{H} 4$. Hydrogen bonds are shown as dashed lines. [Symmetry codes: (i) $1-x$, $1-y,-z$; (vi) $1+x, y, z$; (vii) $1-x, 1-y, 1-z$.] 


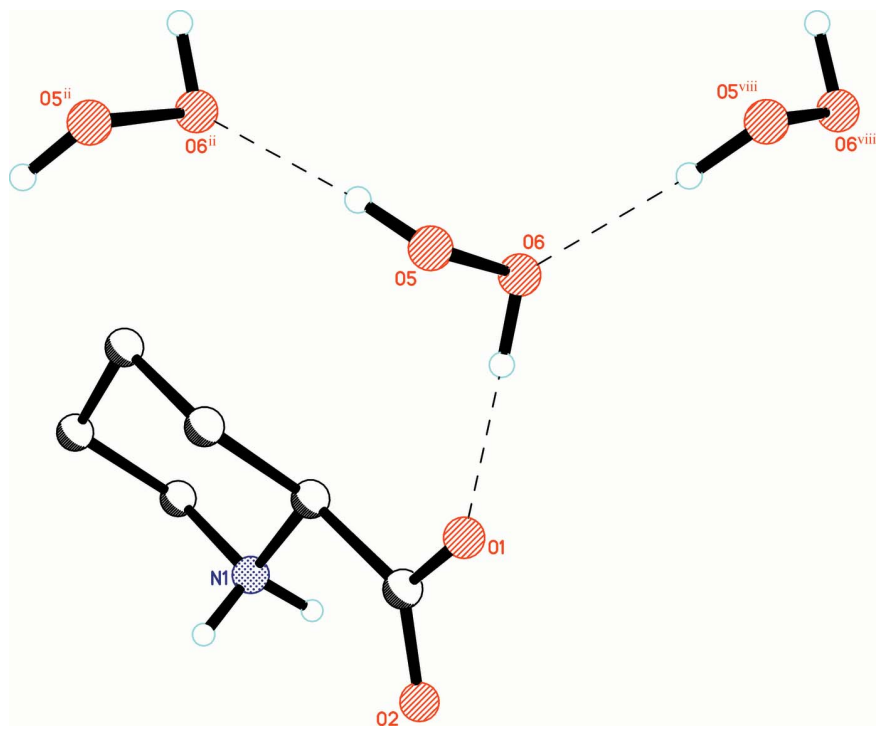

Figure 4

Hydrogen bonds formed by peroxide molecule H5-O5-O6-H6. Hydrogen bonds are shown as dashed lines. [Symmetry codes: (ii) $x$, $\frac{3}{2}-y, \frac{1}{2}+z ;\left(\right.$ viii) $x, \frac{3}{2}-y,-\frac{1}{2}+z$.]

compound (I) is no exception. However, the symmetry-independent peroxide molecules in (I) form a different total number of hydrogen bonds: two donor $\mathrm{HOOH} \cdots{ }^{-} \mathrm{O}_{2} \mathrm{C}$ and two acceptor $\mathrm{N}^{+}-\mathrm{H} \cdots \mathrm{OHOH}$ for $\mathrm{H} 3-\mathrm{O} 3-\mathrm{O} 4-\mathrm{H} 4([2,2]$

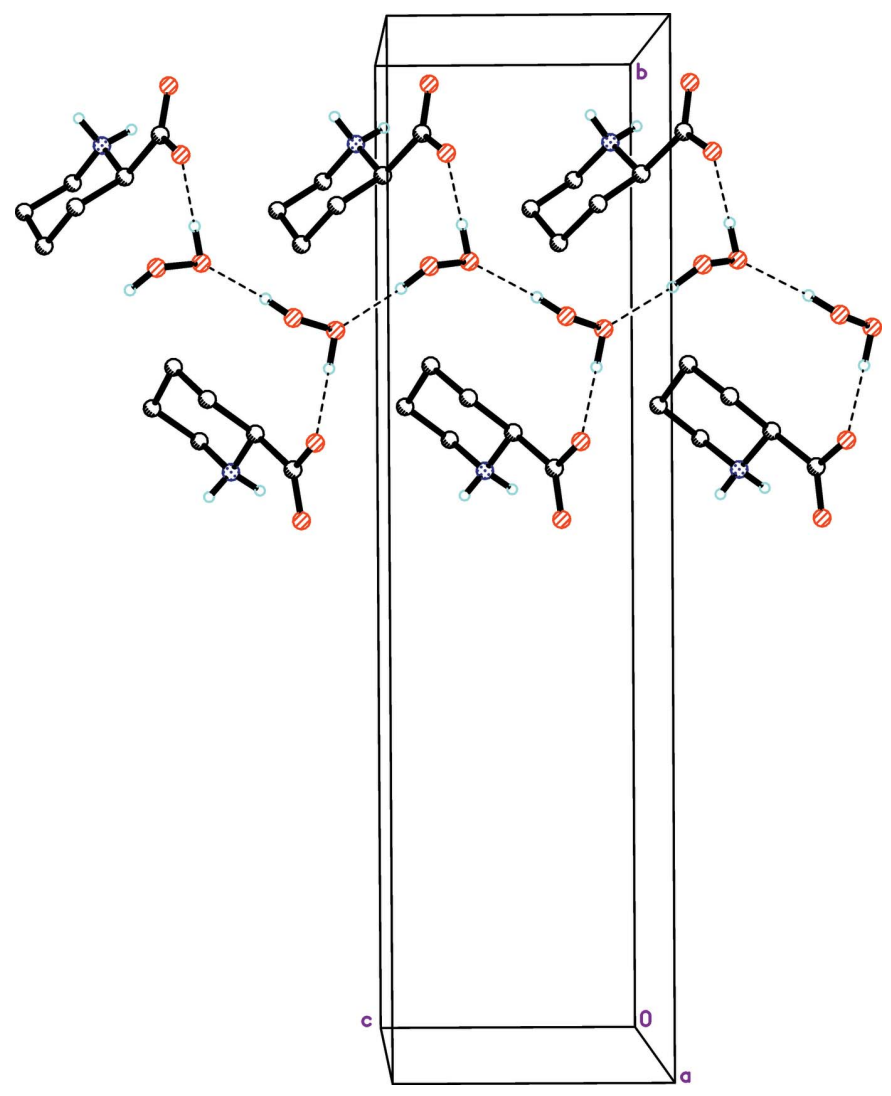

Figure 5

Peroxide hydrogen-bonded chains parallel to the $c$ axis. Hydrogen bonds are shown as dashed lines. mode; Table 1, Fig. 3) and two donor $\mathrm{HOOH} \cdots{ }^{-} \mathrm{O}_{2} \mathrm{C}$ and $\mathrm{HOOH} \cdots \mathrm{OHOH}$ together with one acceptor for $\mathrm{H} 5-\mathrm{O} 5-$ O6-H6 ([2,1] mode; Table 1, Fig. 4). The occurrence of interperoxide hydrogen-bonds results in the formation of simple infinite hydrogen-bonded 'hydroperoxo'-linked chains (Grishanov et al., 2017), running along the $c$-axis direction (Fig. 5). It is significant that such chains and $\mathrm{HOOH}$...OHOH hydrogen bonds were not observed previously in the structures of aliphatic $\alpha$-amino acid peroxosolvates. The reason for this is that charge-assisted $\mathrm{HOOH} .{ }^{-} \mathrm{O}_{2} \mathrm{C}$ bonds are energetically preferable to $\mathrm{HOOH}$. . OHOH interactions (Jesus \& Redinha, 2011; Zick \& Geiger, 2018). For example, in (I) the only interperoxide hydrogen-bond $\mathrm{O} 5-\mathrm{H} 5$ - . O6 is noticeably longer $[2.778(1) \AA]$ than the three $\mathrm{HOOH} \cdots{ }^{-} \mathrm{O}_{2} \mathrm{C}$ bonds [2.641 (1)-2.749 (1) А].

\section{Database survey}

Aliphatic $\alpha$-amino acids contain side chains without heteroatoms suitable for hydrogen-bonding. Up to date, six structures of their peroxosolvates are known: monoperoxosolvates of $N$, N-dimethylglycine $\left(\mathrm{C}_{4} \mathrm{H}_{9} \mathrm{NO}_{2}\right.$; Kapustin et al., 2014), $N$ methylglycine (sarcosine) $\left(\mathrm{C}_{3} \mathrm{H}_{7} \mathrm{NO}_{2} ;\right.$ Navasardyan et al., 2017), isoleucine $\left(\mathrm{C}_{6} \mathrm{H}_{13} \mathrm{NO}_{2}\right.$; Prikhodchenko et al., 2011); sesquiperoxosolvates of glycine $\left(\mathrm{C}_{2} \mathrm{H}_{5} \mathrm{NO}_{2}\right)$, DL-2-aminobutyric acid $\left(\mathrm{C}_{4} \mathrm{H}_{9} \mathrm{NO}_{2}\right)$ and L-phenylalanine $\left(\mathrm{C}_{9} \mathrm{H}_{11} \mathrm{NO}_{2}\right.$; Prikhodchenko et al., 2011). In all of these structures, the organic molecules exist as zwitterions and all peroxide hydrogen atoms are involved in charge-assisted hydrogenbonds with the carboxylate groups. All peroxide molecules adopt skew conformations with $\mathrm{H}-\mathrm{O}-\mathrm{O}-\mathrm{H}$ torsion angles varying between 88.6 and $166.3^{\circ}$.

The carboxylate anions possess four $s p^{2}$-hybridized lone electron pairs suitable for hydrogen-bond formation (Fig. 6)

Figure 6

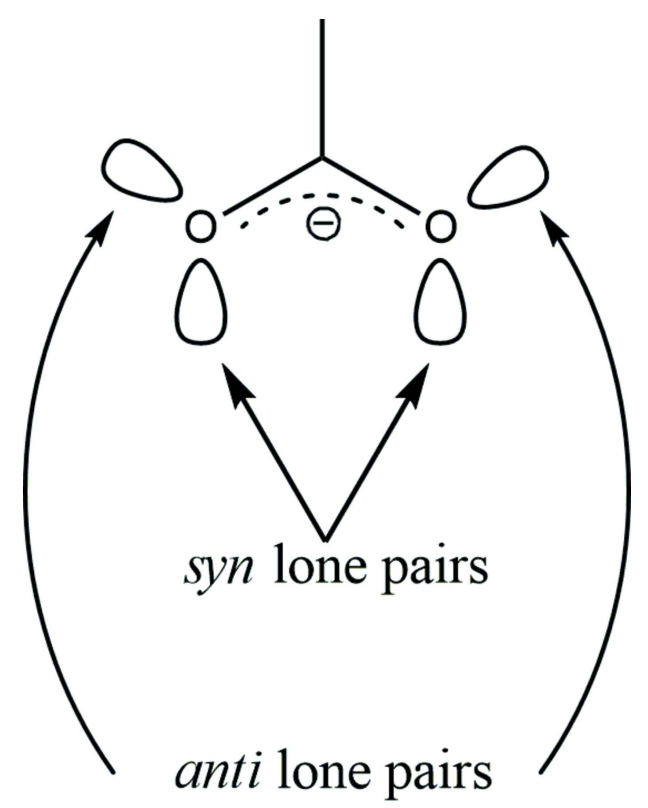

The mutual arrangement of syn and anti lone electron pairs of the carboxylate anion. 
(Mills \& Dean, 1996). It is well known that syn and anti lone pairs exhibit noticeably different basicity (Gandour, 1981; Pal et al., 2018) and hydrogen-bonding properties as a result of electronic and steric effects (Gorbitz \& Etter, 1992; Pranata, 1993). Nine hydrogen-bonded linkage modes are theoretically possible in the structures of amino acid peroxosolvates, taking into account that bifurcated $\mathrm{HOOH} \cdots \mathrm{O}$ bonds are not known (Fig. 7). The two simplest modes $[0 ; \mathrm{S}]$ and $[0 ; \mathrm{A}]$ have not been observed in peroxosolvates of $\alpha$-amino acids, since the peroxide/acid molar ratio is greater than or equal to 1 in each reported structure. The $[\mathrm{S} ; \mathrm{S}]$ linkage was observed in sarcosine monoperoxosolvate. The $[\mathrm{S} ; \mathrm{A}]$ mode was found for the $\mathrm{N}, \mathrm{N}$-dimethylglycine and isoleucine monosolvates. Examples of neither the $[0 ; \mathrm{SA}]$ nor the $[\mathrm{A} ; \mathrm{A}]$ case are currently known. As for three hydrogen bonds, both [S;SA] and [SA;A] linkages were found in the sesquiperoxosolvate structures of glycine, DL-2-aminobutyric acid and L-phenylalanine. Following the same logic, we expected to find [SA;SA] in the structure of the title diperoxosolvate (I). However, the triple hydrogenbonded case [S;SA] occurred, with the fourth donor hydrogen bond $\mathrm{HOOH} \cdots \mathrm{O}$ engaged in forming hydrogen-bonded peroxide chains. It has been shown that the ability of carboxylic anti-orbitals to form hydrogen bonds is strongly affected by steric hindrance caused by $\beta$-substituents in the side chains of carboxylic acids (Gorbitz \& Etter, 1992). It is clear that in (I) the unfeasibility of the fourth carboxylic

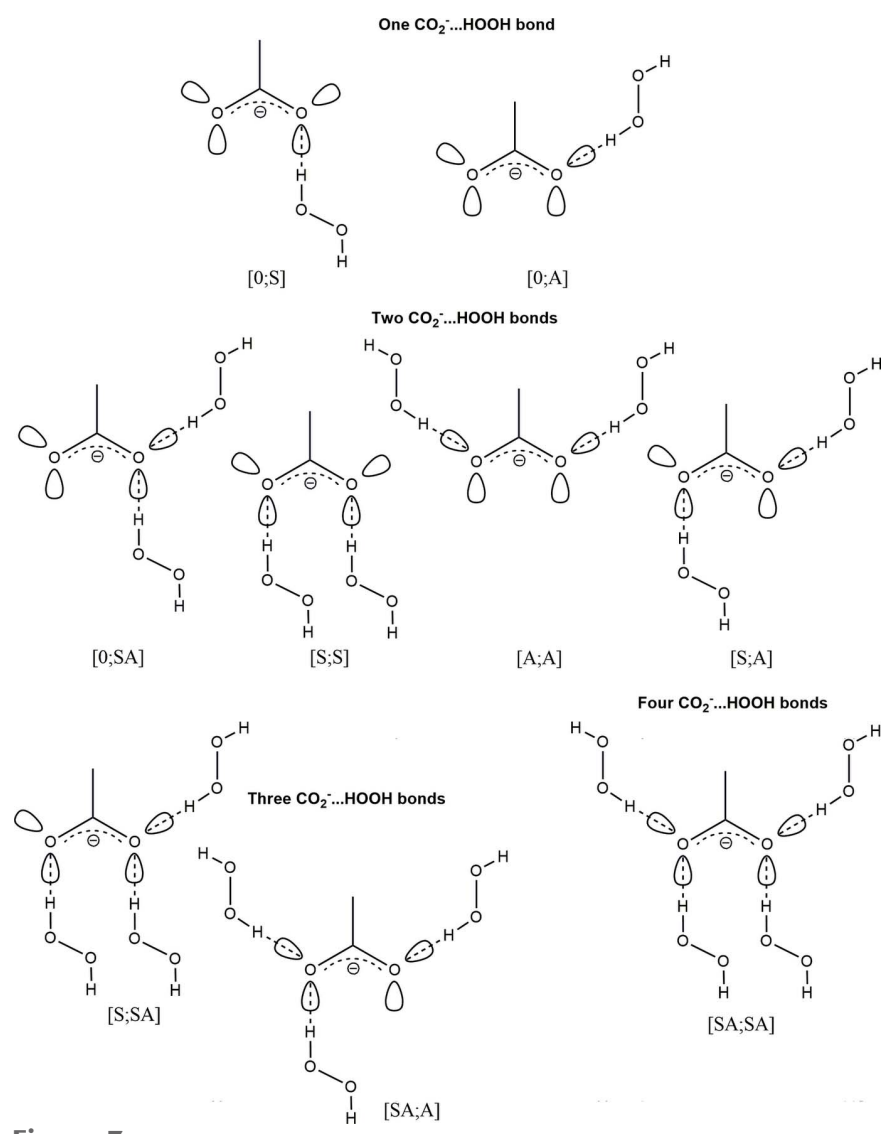

Figure 7

Possible hydrogen-bonded motifs in the structures of amino acid peroxosolvates.
Table 2

Experimental details.

\begin{tabular}{|c|c|}
\hline \multicolumn{2}{|l|}{ Crystal data } \\
\hline Chemical formula & $\mathrm{C}_{6} \mathrm{H}_{11} \mathrm{NO}_{2} \cdot 2 \mathrm{H}_{2} \mathrm{O}_{2}$ \\
\hline$M_{\mathrm{r}}$ & 197.19 \\
\hline Crystal system, space group & Monoclinic, $P 2_{1} / c$ \\
\hline Temperature $(\mathrm{K})$ & 150 \\
\hline$a, b, c(\AA)$ & $6.5739(4), 22.9278(15), 6.0647(4)$ \\
\hline$\beta\left(^{\circ}\right)$ & $93.770(1)$ \\
\hline$V\left(\AA^{3}\right)$ & $912.12(10)$ \\
\hline$Z$ & 4 \\
\hline Radiation type & Мo $K \alpha$ \\
\hline$\mu\left(\mathrm{mm}^{-1}\right)$ & 0.13 \\
\hline Crystal size $(\mathrm{mm})$ & $0.50 \times 0.50 \times 0.50$ \\
\hline \multicolumn{2}{|l|}{ Data collection } \\
\hline Diffractometer & Bruker SMART APEXII \\
\hline Absorption correction & $\begin{array}{l}\text { Multi-scan ( } S A D A B S ; \text { Bruker, } \\
\text { 2008) }\end{array}$ \\
\hline$T_{\min }, T_{\max }$ & $0.659,0.746$ \\
\hline $\begin{array}{l}\text { No. of measured, independent and } \\
\text { observed }[I>2 \sigma(I)] \text { reflections }\end{array}$ & $9809,2418,2170$ \\
\hline$R_{\text {int }}$ & 0.018 \\
\hline$(\sin \theta / \lambda)_{\max }\left(\AA^{-1}\right)$ & 0.682 \\
\hline \multicolumn{2}{|l|}{ Refinement } \\
\hline$R\left[F^{2}>2 \sigma\left(F^{2}\right)\right], w R\left(F^{2}\right), S$ & $0.031,0.084,1.08$ \\
\hline No. of reflections & 2418 \\
\hline No. of parameters & 178 \\
\hline $\mathrm{H}$-atom treatment & All $\mathrm{H}$-atom parameters refined \\
\hline$\Delta \rho_{\max }, \Delta \rho_{\min }\left(\mathrm{e} \AA^{-3}\right)$ & $0.44,-0.19$ \\
\hline
\end{tabular}

Computer programs: APEX2 and SAINT (Bruker, 2008), SHELXL2018/3 (Sheldrick, 2015) and $X P$ in SHELXTL (Sheldrick, 2008).

hydrogen bond is the result of steric effects caused by the peroxide molecules hydrogen bonded with the ammonium group (Fig. 2). It should be noted that the spatial arrangement of the endocyclic amino group in (I) is predefined by the aforementioned planarity of the $\mathrm{N}-\mathrm{C}-\mathrm{CO}_{2}$ amino acid fragment.

\section{Synthesis and crystallization}

96\% Hydrogen peroxide was prepared by an extraction method from serine peroxosolvate (Wolanov et al., 2010). Colourless prismatic crystals of the title compound were obtained by cooling a saturated solution (r.t.) of pipecolinic acid (Aldrich) in $96 \%$ hydrogen peroxide to $255 \mathrm{~K}$. Handling procedures for concentrated hydrogen peroxide have been described in detail (danger of explosion!) by Schumb et al. (1955).

\section{Refinement}

Crystal data, data collection and structure refinement details are summarized in Table 2. All hydrogen atoms were found in difference-Fourier maps and were refined with independent positional and isotropic displacement parameters.

\section{Acknowledgements}

X-ray diffraction studies were performed at the Centre of Shared Equipment of IGIC RAS. 


\section{Funding information}

Funding for this research was provided by: Russian Foundation for Basic Research (award No. 20-03-00449).

\section{References}

Bhattacharjee, S. K. \& Chacko, K. K. (1979). Acta Cryst. B35, 396398.

Bruker (2008). APEX2, SADABS and SAINT. Bruker AXS Inc., Madison, Wisconsin, USA.

Chernyshov, I. Yu., Vener, M. V., Prikhodchenko, P. V., Medvedev, A. G., Lev, O. \& Churakov, A. V. (2017). Cryst. Growth Des. 17, 214 220.

Cronin, D. J., Zhang, X., Bartley, J. P. \& Doherty, W. O. S. (2017). ACS Sustainable Chem. Eng. 5, 6253-6260.

Gandour, R. D. (1981). Bioorg. Chem. 10, 169-176.

Gorbitz, C. H. \& Etter, M. C. (1992). J. Am. Chem. Soc. 114, 627-631.

Grishanov, D. A., Navasardyan, M. A., Medvedev, A. G., Lev, O., Prikhodchenko, P. V. \& Churakov, A. V. (2017). Angew. Chem. Int. Ed. 56, 15241-15245.

Groom, C. R., Bruno, I. J., Lightfoot, M. P. \& Ward, S. C. (2016). Acta Cryst. B72, 171-179.

Jakob, H., Leininger, S., Lehmann, T., Jacobi, S. \& Gutewort, S. (2012). Ullmann's Encyclopedia of Industrial Chemistry, pp 1-33. Weinheim: Wiley-VCH Verlag GmbH \& Co. KGaA.

Jesus, A. J. L. \& Redinha, J. S. (2011). J. Phys. Chem. A, 115, 14069 14077.

Kapustin, E. A., Minkov, V. S. \& Boldyreva, E. V. (2014). CrystEngComm, 16, 10165-10168.

Li, J.-G., Fan, M., Hua, W., Tian, Y., Chen, L.-G., Sun, Y. \& Bai, M.-Y. (2020). Plant Cell, 32, 984-999.
Lyssenko, K. A., Nelyubina, Y. V., Kostyanovsky, R. G. \& Antipin, M. Yu. (2006). ChemPhysChem, 7, 2453-2455.

Mills, J. E. J. \& Dean, P. M. (1996). J. Comput. Aided Mol. Des. 10, 607-622.

Navasardyan, M. A., Bezzubov, S. I., Kuz'mina, L. G., Prikhodchenko, P. V. \& Churakov, A. V. (2017). Acta Cryst. E73, 1793-1796.

Navasardyan, M. A., Grishanov, D. A., Tripol'skaya, T. A., Kuz'mina, L. G., Prikhodchenko, P. V. \& Churakov, A. V. (2018). CrystEngComm, 20, 7413-7416.

Pal, R., Reddy, M., Dinesh, B., Venkatesha, M., Grabowsky, S., Jelsch, C. \& Guru Row, T. N. (2018). J. Phys. Chem. A, 122, 3665-3679.

Pranata, J. (1993). J. Comput. Chem. 14, 685-690.

Prikhodchenko, P. V., Medvedev, A. G., Tripol'skaya, T. A., Churakov, A. V., Wolanov, Y., Howard, J. A. K. \& Lev, O. (2011). CrystEngComm, 13, 2399-2407.

Schumb, W. C., Satterfield, C. N. \& Wentworth, R. P. (1955). Hydrogen peroxide. New York: Reinhold Publishing Corp.

Sheldrick, G. M. (2008). Acta Cryst. A64, 112-122.

Sheldrick, G. M. (2015). Acta Cryst. C71, 3-8.

Stapleton, C. P. D. \& Tiekink, E. R. T. (2001). Acta Cryst. E57, o75o76.

To, E. E., O’Leary, J. J., O’Neill, L. A. J., Vlahos, R., Bozinovski, S., Porter, C. J. H., Brooks, R. D., Brooks, D. A. \& Selemidis, S. (2020). Antioxid. \& Redox Signal. 32, 982-992.

Varadaraj, K. \& Kumari, S. S. (2020). Biochem. Biophys. Res. Commun. 524, 1025-1029.

Wang, H., Schoebel, S., Schmitz, F., Dong, H. \& Hedfalk, K. (2020). Biochim. Biophys. Acta, 1862, 183065.

Wolanov, Y., Lev, O., Churakov, A. V., Medvedev, A. G., Novotortsev, V. M. \& Prikhodchenko, P. V. (2010). Tetrahedron, 66, 51305133.

Zick, P. L. \& Geiger, D. K. (2018). Acta Cryst. C74, 1725-1731. 


\section{supporting information}

Acta Cryst. (2020). E76, 1331-1335 [https://doi.org/10.1107/S205698902000972X]

DL-Piperidinium-2-carboxylate bis(hydrogen peroxide): unusual hydrogenbonded peroxide chains

Mger A. Navasardyan, Dmitry A. Grishanov, Petr V. Prikhodchenko and Andrei V. Churakov

Computing details

Data collection: APEX2 (Bruker, 2008); cell refinement: SAINT (Bruker, 2008); data reduction: SAINT (Bruker, 2008); program(s) used to solve structure: SHELXTL (Sheldrick, 2008); program(s) used to refine structure: SHELXL2018/3 (Sheldrick, 2015); molecular graphics: XP in SHELXTL (Sheldrick, 2008).

DL-Piperidinium-2-carboxylate bis(hydrogen peroxide)

Crystal data

$\mathrm{C}_{6} \mathrm{H}_{11} \mathrm{NO}_{2} \cdot 2 \mathrm{H}_{2} \mathrm{O}_{2}$

$M_{r}=197.19$

Monoclinic, $P 2{ }_{1} / c$

$a=6.5739(4) \AA$

$b=22.9278(15) \AA$

$c=6.0647$ (4) $\AA$

$\beta=93.770(1)^{\circ}$

$V=912.12(10) \AA^{3}$

$Z=4$

Data collection

Bruker SMART APEXII

diffractometer

$\omega$ scans

Absorption correction: multi-scan

(SADABS; Bruker, 2008)

$T_{\min }=0.659, T_{\max }=0.746$

9809 measured reflections

\section{Refinement}

Refinement on $F^{2}$

Least-squares matrix: full

$R\left[F^{2}>2 \sigma\left(F^{2}\right)\right]=0.031$

$w R\left(F^{2}\right)=0.084$

$S=1.08$

2418 reflections

178 parameters

0 restraints

Primary atom site location: structure-invariant direct methods
$F(000)=424$

$D_{\mathrm{x}}=1.436 \mathrm{Mg} \mathrm{m}^{-3}$

Mo $K \alpha$ radiation, $\lambda=0.71073 \AA$

Cell parameters from 5232 reflections

$\theta=3.1-30.6^{\circ}$

$\mu=0.13 \mathrm{~mm}^{-1}$

$T=150 \mathrm{~K}$

Nugget, colourless

$0.50 \times 0.50 \times 0.50 \mathrm{~mm}$

2418 independent reflections

2170 reflections with $I>2 \sigma(I)$

$R_{\text {int }}=0.018$

$\theta_{\text {max }}=29.0^{\circ}, \theta_{\min }=3.1^{\circ}$

$h=-8 \rightarrow 8$

$k=-30 \rightarrow 31$

$l=-8 \rightarrow 8$

Secondary atom site location: difference Fourier map

Hydrogen site location: difference Fourier map

All $\mathrm{H}$-atom parameters refined

$w=1 /\left[\sigma^{2}\left(F_{0}^{2}\right)+(0.0419 P)^{2}+0.218 P\right]$

where $P=\left(F_{\mathrm{o}}^{2}+2 F_{\mathrm{c}}{ }^{2}\right) / 3$

$(\Delta / \sigma)_{\max }=0.001$

$\Delta \rho_{\max }=0.44 \mathrm{e} \AA^{-3}$

$\Delta \rho_{\min }=-0.19$ e $\AA^{-3}$ 


\section{Special details}

Geometry. All esds (except the esd in the dihedral angle between two 1.s. planes) are estimated using the full covariance matrix. The cell esds are taken into account individually in the estimation of esds in distances, angles and torsion angles; correlations between esds in cell parameters are only used when they are defined by crystal symmetry. An approximate (isotropic) treatment of cell esds is used for estimating esds involving l.s. planes.

Fractional atomic coordinates and isotropic or equivalent isotropic displacement parameters $\left(\AA^{2}\right)$

\begin{tabular}{lllll}
\hline & $x$ & $y$ & $z$ & $U_{\text {iso }} * / U_{\text {eq }}$ \\
\hline N1 & $-0.02450(12)$ & $0.57964(3)$ & $0.57611(13)$ & $0.01528(16)$ \\
H1 & $-0.093(2)$ & $0.5604(6)$ & $0.463(2)$ & $0.029(3)^{*}$ \\
H2 & $0.039(2)$ & $0.5538(6)$ & $0.661(2)$ & $0.025(3)^{*}$ \\
O1 & $0.40875(11)$ & $0.60608(3)$ & $0.25095(12)$ & $0.02266(17)$ \\
O2 & $0.21298(11)$ & $0.52860(3)$ & $0.30218(11)$ & $0.02005(16)$ \\
C1 & $-0.17311(14)$ & $0.61336(4)$ & $0.70359(15)$ & $0.01777(18)$ \\
H11 & $-0.2642(19)$ & $0.5851(6)$ & $0.759(2)$ & $0.021(3)^{*}$ \\
H12 & $-0.2449(19)$ & $0.6384(6)$ & $0.597(2)$ & $0.024(3)^{*}$ \\
C2 & $-0.05768(15)$ & $0.64708(4)$ & $0.88702(15)$ & $0.01993(19)$ \\
H21 & $-0.157(2)$ & $0.6679(6)$ & $0.968(2)$ & $0.029(3)^{*}$ \\
H22 & $0.010(2)$ & $0.6192(6)$ & $0.988(2)$ & $0.029(3)^{*}$ \\
C3 & $0.10082(16)$ & $0.68727(4)$ & $0.79508(17)$ & $0.0218(2)$ \\
H32 & $0.033(2)$ & $0.7172(6)$ & $0.703(2)$ & $0.027(3)^{*}$ \\
H31 & $0.179(2)$ & $0.7082(6)$ & $0.916(2)$ & $0.030(3)^{*}$ \\
C4 & $0.24769(14)$ & $0.65223(4)$ & $0.66075(16)$ & $0.01923(19)$ \\
H41 & $0.343(2)$ & $0.6784(6)$ & $0.590(2)$ & $0.027(3)^{*}$ \\
H42 & $0.326(2)$ & $0.6256(6)$ & $0.759(2)$ & $0.027(3)^{*}$ \\
C5 & $0.13006(13)$ & $0.61800(4)$ & $0.47837(14)$ & $0.01483(17)$ \\
H52 & $0.0520(18)$ & $0.6451(5)$ & $0.377(2)$ & $0.019(3)^{*}$ \\
C6 & $0.26188(13)$ & $0.58042(4)$ & $0.33422(14)$ & $0.01570(18)$ \\
O3 & $0.74598(11)$ & $0.54761(3)$ & $0.18291(11)$ & $0.01986(16)$ \\
O4 & $0.72361(11)$ & $0.48529(3)$ & $0.13828(12)$ & $0.02162(16)$ \\
H4 & $0.737(2)$ & $0.4839(7)$ & $-0.003(3)$ & $0.040(4)^{*}$ \\
H3 & $0.620(3)$ & $0.5583(7)$ & $0.194(3)$ & $0.040(4)^{*}$ \\
O5 & $0.67290(12)$ & $0.72538(4)$ & $0.33260(14)$ & $0.02787(18)$ \\
O6 & $0.49801(12)$ & $0.71623(3)$ & $0.17405(13)$ & $0.02507(17)$ \\
H5 & $0.613(2)$ & $0.7454(7)$ & $0.434(3)$ & $0.041(4)^{*}$ \\
H6 & $0.472(2)$ & $0.6792(7)$ & $0.199(2)$ & $0.034(4)^{*}$ \\
& & & &
\end{tabular}

Atomic displacement parameters $\left(\AA^{2}\right)$

\begin{tabular}{lllllll}
\hline & $U^{11}$ & $U^{22}$ & $U^{33}$ & $U^{12}$ & $U^{13}$ & $U^{23}$ \\
\hline $\mathrm{N} 1$ & $0.0163(3)$ & $0.0143(4)$ & $0.0153(3)$ & $-0.0005(3)$ & $0.0025(3)$ & $-0.0015(3)$ \\
$\mathrm{O} 1$ & $0.0229(4)$ & $0.0170(3)$ & $0.0293(4)$ & $-0.0015(3)$ & $0.0110(3)$ & $-0.0023(3)$ \\
$\mathrm{O} 2$ & $0.0237(3)$ & $0.0149(3)$ & $0.0221(3)$ & $-0.0016(2)$ & $0.0060(3)$ & $-0.0044(2)$ \\
$\mathrm{C} 1$ & $0.0169(4)$ & $0.0183(4)$ & $0.0185(4)$ & $0.0023(3)$ & $0.0044(3)$ & $-0.0002(3)$ \\
$\mathrm{C} 2$ & $0.0237(5)$ & $0.0202(4)$ & $0.0163(4)$ & $0.0037(4)$ & $0.0042(3)$ & $-0.0023(3)$ \\
$\mathrm{C} 3$ & $0.0251(5)$ & $0.0179(4)$ & $0.0225(5)$ & $-0.0009(4)$ & $0.0036(4)$ & $-0.0074(4)$ \\
$\mathrm{C} 4$ & $0.0186(4)$ & $0.0182(4)$ & $0.0211(4)$ & $-0.0024(3)$ & $0.0023(3)$ & $-0.0057(3)$
\end{tabular}




\begin{tabular}{lllllll} 
C5 & $0.0165(4)$ & $0.0132(4)$ & $0.0150(4)$ & $-0.0002(3)$ & $0.0028(3)$ & $-0.0004(3)$ \\
C6 & $0.0170(4)$ & $0.0162(4)$ & $0.0140(4)$ & $0.0019(3)$ & $0.0018(3)$ & $-0.0012(3)$ \\
O3 & $0.0193(3)$ & $0.0172(3)$ & $0.0229(3)$ & $0.0006(3)$ & $0.0008(3)$ & $-0.0044(2)$ \\
O4 & $0.0301(4)$ & $0.0158(3)$ & $0.0187(3)$ & $0.0029(3)$ & $0.0003(3)$ & $-0.0012(2)$ \\
O5 & $0.0261(4)$ & $0.0308(4)$ & $0.0265(4)$ & $-0.0044(3)$ & $0.0001(3)$ & $-0.0053(3)$ \\
O6 & $0.0308(4)$ & $0.0200(4)$ & $0.0237(4)$ & $-0.0027(3)$ & $-0.0029(3)$ & $0.0024(3)$ \\
\hline
\end{tabular}

Geometric parameters $\left(\AA,{ }^{\circ}\right)$

\begin{tabular}{|c|c|c|c|}
\hline $\mathrm{N} 1-\mathrm{C} 5$ & $1.4955(11)$ & $\mathrm{C} 3-\mathrm{H} 32$ & $0.974(14)$ \\
\hline $\mathrm{N} 1-\mathrm{C} 1$ & $1.4996(11)$ & $\mathrm{C} 3-\mathrm{H} 31$ & $0.991(14)$ \\
\hline $\mathrm{N} 1-\mathrm{H} 1$ & $0.912(15)$ & $\mathrm{C} 4-\mathrm{C} 5$ & $1.5247(12)$ \\
\hline $\mathrm{N} 1-\mathrm{H} 2$ & $0.875(15)$ & $\mathrm{C} 4-\mathrm{H} 41$ & $0.986(14)$ \\
\hline $\mathrm{O} 1-\mathrm{C} 6$ & $1.2639(11)$ & $\mathrm{C} 4-\mathrm{H} 42$ & $0.975(14)$ \\
\hline $\mathrm{O} 2-\mathrm{C} 6$ & $1.2429(11)$ & $\mathrm{C} 5-\mathrm{C} 6$ & $1.5353(12)$ \\
\hline $\mathrm{C} 1-\mathrm{C} 2$ & $1.5166(13)$ & $\mathrm{C} 5-\mathrm{H} 52$ & $0.991(12)$ \\
\hline $\mathrm{C} 1-\mathrm{H} 11$ & $0.958(13)$ & $\mathrm{O} 3-\mathrm{O} 4$ & $1.4600(9)$ \\
\hline $\mathrm{C} 1-\mathrm{H} 12$ & $0.966(13)$ & $\mathrm{O} 3-\mathrm{H} 3$ & $0.872(17)$ \\
\hline $\mathrm{C} 2-\mathrm{C} 3$ & $1.5239(14)$ & $\mathrm{O} 4-\mathrm{H} 4$ & $0.866(18)$ \\
\hline $\mathrm{C} 2-\mathrm{H} 21$ & $0.968(14)$ & $\mathrm{O} 5-\mathrm{O} 6$ & $1.4646(11)$ \\
\hline $\mathrm{C} 2-\mathrm{H} 22$ & $0.972(14)$ & $\mathrm{O} 5-\mathrm{H} 5$ & $0.883(17)$ \\
\hline $\mathrm{C} 3-\mathrm{C} 4$ & $1.5313(13)$ & O6- $-\mathrm{H} 6$ & $0.881(16)$ \\
\hline $\mathrm{C} 5-\mathrm{N} 1-\mathrm{C} 1$ & $112.57(7)$ & $\mathrm{C} 4-\mathrm{C} 3-\mathrm{H} 31$ & $109.3(8)$ \\
\hline $\mathrm{C} 5-\mathrm{N} 1-\mathrm{H} 1$ & $107.6(9)$ & $\mathrm{H} 32-\mathrm{C} 3-\mathrm{H} 31$ & $106.3(11)$ \\
\hline $\mathrm{C} 1-\mathrm{N} 1-\mathrm{H} 1$ & $109.2(9)$ & $\mathrm{C} 5-\mathrm{C} 4-\mathrm{C} 3$ & $110.40(8)$ \\
\hline $\mathrm{C} 5-\mathrm{N} 1-\mathrm{H} 2$ & $108.7(9)$ & $\mathrm{C} 5-\mathrm{C} 4-\mathrm{H} 41$ & $107.7(8)$ \\
\hline $\mathrm{C} 1-\mathrm{N} 1-\mathrm{H} 2$ & $110.4(9)$ & $\mathrm{C} 3-\mathrm{C} 4-\mathrm{H} 41$ & $110.7(8)$ \\
\hline $\mathrm{H} 1-\mathrm{N} 1-\mathrm{H} 2$ & $108.2(12)$ & $\mathrm{C} 5-\mathrm{C} 4-\mathrm{H} 42$ & $110.0(8)$ \\
\hline $\mathrm{N} 1-\mathrm{C} 1-\mathrm{C} 2$ & $109.24(7)$ & $\mathrm{C} 3-\mathrm{C} 4-\mathrm{H} 42$ & $109.4(8)$ \\
\hline $\mathrm{N} 1-\mathrm{C} 1-\mathrm{H} 11$ & $106.0(8)$ & $\mathrm{H} 41-\mathrm{C} 4-\mathrm{H} 42$ & $108.6(11)$ \\
\hline $\mathrm{C} 2-\mathrm{C} 1-\mathrm{H} 11$ & $112.4(8)$ & $\mathrm{N} 1-\mathrm{C} 5-\mathrm{C} 4$ & $109.91(7)$ \\
\hline $\mathrm{N} 1-\mathrm{C} 1-\mathrm{H} 12$ & $105.4(8)$ & $\mathrm{N} 1-\mathrm{C} 5-\mathrm{C} 6$ & $108.62(7)$ \\
\hline $\mathrm{C} 2-\mathrm{C} 1-\mathrm{H} 12$ & $112.9(8)$ & $\mathrm{C} 4-\mathrm{C} 5-\mathrm{C} 6$ & $115.06(7)$ \\
\hline $\mathrm{H} 11-\mathrm{C} 1-\mathrm{H} 12$ & $110.4(11)$ & $\mathrm{N} 1-\mathrm{C} 5-\mathrm{H} 52$ & $106.0(7)$ \\
\hline $\mathrm{C} 1-\mathrm{C} 2-\mathrm{C} 3$ & $111.17(8)$ & $\mathrm{C} 4-\mathrm{C} 5-\mathrm{H} 52$ & $110.1(7)$ \\
\hline $\mathrm{C} 1-\mathrm{C} 2-\mathrm{H} 21$ & $107.6(8)$ & $\mathrm{C} 6-\mathrm{C} 5-\mathrm{H} 52$ & $106.7(7)$ \\
\hline $\mathrm{C} 3-\mathrm{C} 2-\mathrm{H} 21$ & $112.8(8)$ & $\mathrm{O} 2-\mathrm{C} 6-\mathrm{O} 1$ & $125.38(8)$ \\
\hline $\mathrm{C} 1-\mathrm{C} 2-\mathrm{H} 22$ & $108.3(8)$ & $\mathrm{O} 2-\mathrm{C} 6-\mathrm{C} 5$ & $118.40(8)$ \\
\hline $\mathrm{C} 3-\mathrm{C} 2-\mathrm{H} 22$ & $109.6(8)$ & $\mathrm{O} 1-\mathrm{C} 6-\mathrm{C} 5$ & $116.16(8)$ \\
\hline $\mathrm{H} 21-\mathrm{C} 2-\mathrm{H} 22$ & $107.3(11)$ & $\mathrm{O} 4-\mathrm{O} 3-\mathrm{H} 3$ & $101.8(11)$ \\
\hline $\mathrm{C} 2-\mathrm{C} 3-\mathrm{C} 4$ & $110.34(8)$ & $\mathrm{O} 3-\mathrm{O} 4-\mathrm{H} 4$ & $101.7(10)$ \\
\hline $\mathrm{C} 2-\mathrm{C} 3-\mathrm{H} 32$ & $109.7(8)$ & $\mathrm{O} 6-\mathrm{O} 5-\mathrm{H} 5$ & $99.6(11)$ \\
\hline $\mathrm{C} 4-\mathrm{C} 3-\mathrm{H} 32$ & $110.2(8)$ & $\mathrm{O} 5-\mathrm{O} 6-\mathrm{H} 6$ & $100.4(10)$ \\
\hline $\mathrm{C} 2-\mathrm{C} 3-\mathrm{H} 31$ & $111.0(8)$ & & \\
\hline $\mathrm{C} 5-\mathrm{N} 1-\mathrm{C} 1-\mathrm{C} 2$ & $-58.48(10)$ & $\mathrm{C} 3-\mathrm{C} 4-\mathrm{C} 5-\mathrm{C} 6$ & $-179.22(8)$ \\
\hline $\mathrm{N} 1-\mathrm{C} 1-\mathrm{C} 2-\mathrm{C} 3$ & $56.95(10)$ & $\mathrm{N} 1-\mathrm{C} 5-\mathrm{C} 6-\mathrm{O} 2$ & $6.85(11)$ \\
\hline
\end{tabular}


supporting information

$\begin{array}{llll}\mathrm{C} 1-\mathrm{C} 2-\mathrm{C} 3-\mathrm{C} 4 & -56.65(11) & \mathrm{C} 4-\mathrm{C} 5-\mathrm{C} 6-\mathrm{O} 2 & 130.51(9) \\ \mathrm{C} 2-\mathrm{C} 3-\mathrm{C} 4-\mathrm{C} 5 & 55.85(11) & \mathrm{N} 1-\mathrm{C} 5-\mathrm{C} 6-\mathrm{O} 1 & -175.64(8) \\ \mathrm{C} 1-\mathrm{N} 1-\mathrm{C} 5-\mathrm{C} 4 & 58.51(10) & \mathrm{C} 4-\mathrm{C} 5-\mathrm{C} 6-\mathrm{O} 1 & -51.98(11) \\ \mathrm{C} 1-\mathrm{N} 1-\mathrm{C} 5-\mathrm{C} 6 & -174.80(7) & \mathrm{H} 3-\mathrm{O} 3-\mathrm{O} 4-\mathrm{H} 4 & 102.5(15) \\ \mathrm{C} 3-\mathrm{C} 4-\mathrm{C} 5-\mathrm{N} 1 & -56.25(10) & \mathrm{H} 5-\mathrm{O} 5-\mathrm{O} 6-\mathrm{H} 6 & -105.1(15)\end{array}$

Hydrogen-bond geometry $\left(\AA,{ }^{\circ}\right)$

\begin{tabular}{lllll}
\hline$D-\mathrm{H} \cdots A$ & $D-\mathrm{H}$ & $\mathrm{H} \cdots A$ & $D \cdots A$ & $D-\mathrm{H} \cdots A$ \\
\hline $\mathrm{O} 3-\mathrm{H} 3 \cdots \mathrm{O} 1$ & $0.872(17)$ & $1.819(17)$ & $2.6463(10)$ & $157.8(15)$ \\
$\mathrm{O} 4-\mathrm{H} 4 \cdots \mathrm{O} 2^{\mathrm{i}}$ & $0.866(18)$ & $1.889(18)$ & $2.7490(10)$ & $172.2(15)$ \\
$\mathrm{O} 6-\mathrm{H} 6 \cdots \mathrm{O} 1$ & $0.881(16)$ & $1.760(16)$ & $2.6412(10)$ & $177.4(14)$ \\
$\mathrm{O} 5-\mathrm{H} 5 \cdots 6^{\mathrm{ii}}$ & $0.883(17)$ & $1.898(18)$ & $2.7777(12)$ & $174.4(15)$ \\
$\mathrm{N} 1-\mathrm{H} 1 \cdots{ }^{\mathrm{iii}}$ & $0.912(15)$ & $1.961(15)$ & $2.8336(11)$ & $159.6(13)$ \\
$\mathrm{N} 1-\mathrm{H} 2 \cdots{ }^{i v}$ & $0.875(15)$ & $2.112(15)$ & $2.9459(11)$ & $159.1(12)$
\end{tabular}

Symmetry codes: (i) $-x+1,-y+1,-z$; (ii) $x,-y+3 / 2, z+1 / 2$; (iii) $x-1, y, z$; (iv) $-x+1,-y+1,-z+1$. 